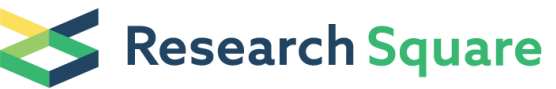 \\ Preprints are preliminary reports that have not undergone peer review. They should not be considered conclusive, used to inform clinical practice, or referenced by the media as validated information.
}

\section{Kanglexin promotes diabetic wound healing through activating FGFR/ERK1/2 signaling pathway mediated angiogenesis.}

\section{Zhao Yixiu}

Harbin Medical University

\section{Xinhui Wang}

State-Province Key Laboratory of Biomedicine-Pharmaceutics of China, Key Laboratory of Cardiovascular Medicine Research, Ministry of Education, Department of Pharmacology, College of Pharmacy

\section{Shuang Yang}

State-Province Key Laboratory of Biomedicine-Pharmaceutics of China, Key Laboratory of Cardiovascular Medicine Research, Ministry of Education, Department of Pharmacology, College of Pharmacy

\section{Xia Song}

State-Province Key Laboratory of Biomedicine-Pharmaceutics of China, Key Laboratory of Cardiovascular Medicine Research, Ministry of Education, Department of Pharmacology, College of Pharmacy

\section{Chao Chen}

State-Province Key Laboratory of Biomedicine-Pharmaceutics of China, Key Laboratory of Cardiovascular Medicine Research, Ministry of Education, Department of Pharmacology, College of Pharmacy

\section{Na Sun}

State-Province Key Laboratory of Biomedicine-Pharmaceutics of China, Key Laboratory of Cardiovascular Medicine Research, Ministry of Education, Department of Pharmacology, College of Pharmacy

\section{Yannan Zhang}

State-Province Key Laboratory of Biomedicine-Pharmaceutics of China, Key Laboratory of Cardiovascular Medicine Research, Ministry of Education, Department of Pharmacology, College of Pharmacy

\section{Dahong Yao}

Department of Medicinal Chemistry and Natural Medicine Chemistry, College of Pharmacy

\section{Jian Huang}

Department of Medicinal Chemistry and Natural Medicine Chemistry, College of Pharmacy Jinhui Wang 
Department of Medicinal Chemistry and Natural Medicine Chemistry, College of Pharmacy

\section{Yan Zhang ( $\square$ zhangyan@ems.hrbmu.edu.cn )}

State-Province Key Laboratory of Biomedicine-Pharmaceutics of China, Key Laboratory of Cardiovascular Medicine Research, Ministry of Education, Department of Pharmacology, College of Pharmacy

\section{Baofeng Yang ( $\nabla$ yangbf@ems.hrbmu.edu.cn )}

State-Province Key Laboratory of Biomedicine-Pharmaceutics of China, Key Laboratory of Cardiovascular Medicine Research, Ministry of Education, Department of Pharmacology, College of Pharmacy, Harbin Medical University, Harbin, China

\section{Research}

Keywords: diabetic wounds; FGFR; ERK1/2; AGEs; angiogenesis

Posted Date: May 15th, 2020

DOI: https://doi.org/10.21203/rs.3.rs-28087/v1

License: (c) (1) This work is licensed under a Creative Commons Attribution 4.0 International License. Read Full License 


\section{Abstract}

Background: Diabetic wounds (DWs), especially leg or foot ulcers, are common diabetic complications, accounting for about $20 \%$ of diabetic patients. At present, there is still lack of effective measures to treat DWs in clinical practice. Kanglexin (KLX) is an anthraquinone compound with vasoprotective effect. This study will explore the therapeutic effects of KLX on DWs and the underlying mechanisms.

Method: KM mice were injected with streptozocin $(180 \mathrm{mg} / \mathrm{kg})$ intraperitoneally to establish animal model of Type 1 Diabetes. The full-thickness skin wounds with the diameter of $5 \mathrm{~mm}$ were prepared on the back of diabetic mice with a skin punch. KLX is dissolved in sterile injection oil and applied to the wound once a day for 14 consecutive days. The wounds were photographed every day and the wound size was analyzed by Image Pro Plus software. On the $3^{\text {rd }}, 7^{\text {th }}, 11^{\text {th }}$ day after $K L X$ administration, skin tissues with a diameter of $1 \mathrm{~cm}$ around the wound were fixed in paraformaldehyde and subjected to $\mathrm{HE}$, Masson and immunohistochemical staining. Collagen deposition were evaluated by hydroxyproline kit. Angiogenesis of wound skin was evaluated by recording the formation of new blood vessels on its subsurface. In vitro study, human umbilical vein endothelial cells (HUVECs) were treated with advanced glycation end products (AGEs, $100 \mu \mathrm{g} / \mathrm{mL}$ ) to establish the cell model of DWs. The proliferation, migration and tubular structure formation of HUVECs were measured by MTT, scratch and tubule formation experiments, respectively. Phosphorylation of ERK1/2 was detected by Western blot analysis.

Results

Firstly, in-vivo study showed that KLX significantly accelerated the closure of diabetic wounds through promoting granulation tissue formation, collagen synthesis and angiogenesis. Secondly, in vitro study results further confirmed that KLX promoted the proliferation, migration and tubular structure formation of HUVECs. Besides, KLX significantly up-regulated phospho-ERK1/2 both in diabetic wounds and AGEstreated HUVECs. Thirdly, molecular docking simulation results indicated that there were multiple hydrogen bonds between KLX and FGFR amino acid residues, and FGFR inhibitor PD173074 significantly reversed KLX's promotion of ERK1/2 phosphorylation and angiogenesis.

\section{Conclusions:}

KLX promotes angiogenesis and accelerates the healing of diabetic wounds by activating FGFR and ERK1/2 signaling pathway.

\section{Background}

Diabetes mellitus, characterized by chronic hyperglycemia and metabolic syndrome, has become one of leading causes of death following with cardiovascular disease and cancer, seriously threatening human health. According to data collected by International Diabetes Federation, there were 425 million diabetics all over the world in 2017, and this number was expected to reach 629 million by 2045 [1, 2]. As we all know, long-term hyperglycemia leads to chronic damage of various organs and tissues, especially eyes, 
kidneys, heart, blood vessels and nerve, referred to as diabetic complications. Among people with diabetes, about $30 \sim 80 \%$ are combined with diabetic wounds (DWs), which are manifested as the destruction of foot or lower limb tissues, resulting in ulcers and infections that are difficult to cure, and even lead to amputation $[3,4]$. In addition to the tremendous damage to patient's health and life, DWs also bring about a huge financial burden. Data shows that the cost of treatment and care for diabetic foot without primary ischemic symptoms is $\$ 16,000$. If combined with primary ischemic symptoms, this cost will increase to $\$ 26,700[5,6]$. Therefore, there is an urgent need to address the issues of treatment and care of DWs.

Wound healing is an evolutionarily conservative, complicated, multicellular regulated biological process. Its purpose is to recover the barrier function of the skin [7]. When the skin is injured, the body activates a series of mechanisms to repair the injured tissue, referred to as "healing cascade". The healing cascade can be divided into four stages: hemostasis, inflammation, proliferation, and maturation [8]. Wound healing usually begins with hemostasis and microbial invasion at the wound, initiating the inflammatory phase. During the inflammatory phase, pro-inflammatory cells such as neutrophils and macrophages increase rapidly to remove pathogens and cell debris from the wound. After this, the proliferative phase begins, and new tissues, new matrix and new blood vessels forms gradually to fill the wound area and promote wound healing [9]. However, DWs exhibit a continuous inflammatory stage, accompanied by impaired formation of mature granulation tissue and collagen, leading to a reduction in wound tensile strength. This phenomenon may be caused by microangiopathy and damaged angiogenesis at the wound site, leading to local ischemia and hypoxia $[10,11]$. Angiogenesis is regulated by a variety of angiogenesis-inducing growth factors around the wound. By binding to their receptors, growth factors activate multiple downstream signaling pathways, promote the proliferation, migration, and differentiation of vascular endothelial cells and promote angiogenesis [12, 13]. However, studies have shown that growth factors and their receptors in the wound area have changed both in type and abundance in diabetic patients. The possible reason is that long-term hyperglycemia and oxidative stress increase the formation of advanced glycation end products (AGEs), reduce the activity of growth factors, affect endothelial cell functions, and delay wound healing $[14,15]$.

With the development of biological science and technology, bioengineered drugs such as growth factors, skin substitutes and cytokines have been used to treat DWs. Currently, there are numerous marketed drugs that promote wound healing. However, due to so many risk factors affecting DWs, the cure ratio is still less than $50 \%[16,17]$. The possible reason is that chronic wounds are prone to produce proteolytic environments, which directly degrade biological drugs such as growth factors; or up-regulated proinflammatory factors and chemokines in wound area indirectly inhibit the function of growth factors [1819]. Therefore, there is an urgent need for effective treatment to promote diabetic wound healing. KLX is a compound with the parent nucleus of anthraquinone (Fig. 1). Our previous studies have shown that KLX has vascular endothelium protecting and vascular tension decreasing effect [20]. Since angiogenesis is closely related to vascular endothelial function, we examined the effect of KLX on angiogenesis and DWs and explored the underlying mechanisms. 


\section{Materials And Methods}

\section{Materials}

Streptozocin, Masson Staining Kit were purchased from Solarbio Life Science, Beijing, China; Sterile Soybean Oil for Injection (CAS: 8001-22-7) was purchased from Sheng Rui Medicine, Xi'an, China; Hydroxyproline Assay Kit was purchased from Nanjing Jiancheng Bioengineering Institute, Nanjing, China; HE Staining Kit, Triton X-100, RIPA was purchased from Beyotime Biotechnology, Shanghai, China; a-SMA primary antibody for immunohistochemistry experiment was purchased from Boster Biological Technology, Wuhan, China; secondary antibody for immunohistochemistry experiment was purchased from ZSGB-Bio, Beijing, China; Human umbilical vein endothelial cells (HUVECs) was purchased from Sciencell Research Laboratories (San Diego, CA, USA). Phospho-ERK1/2, $\beta$-actin antibody was purchased from Cell Signaling Technology, Danvers, USA; anti-HRP antibody was purchased from LI-COR Biosciences, USA; MTT was purchased from Sigma-Aldrich, USA; AGEs was purchased from Biovision, San Francisco, USA; Matrigel was purchased from BD Biosciences, Franklin Lakes, USA; Calcein AM, PD173074, ZM306416, AG1296 and PD153035 was purchased from Abcam, Cambridge, UK.

\section{Preparation of drugs}

KLX with a purity of $99 \%$ was provided by department of pharmaceutical chemistry (College of Pharmacy, Harbin Medical University) and dissolved in Sterile Soybean Oil for Injection at the concentration of 30, $100,300 \mu \mathrm{g} / \mathrm{mL}$.

\section{In vivo study}

KM mice (male, 18-20 g, SPF level) were purchased from Laboratory Animal Center of Second Affiliated Hospital of Harbin Medical University. Mice were randomly divided into two groups according to their original body weight: normal mice and diabetic mice. Diabetic mice were intraperitoneally injected with streptozocin $(180 \mathrm{mg} / \mathrm{kg})$ to establish the animal model of Type 1 Diabetes. After 3 days, the fasting glucose was measured. The mice with fasting glucose higher than $11.1 \mathrm{mmol} / \mathrm{L}$ can be used as diabetic mice for subsequent experiments. Diabetic mice were randomly divided into 6 groups according to their fasting glucose level: diabetic wound without treatment (DW), diabetic wound treated with blank oil (DW + Blank oil), diabetic wound treated with $\mathrm{KLX}$ oil $30 \mu \mathrm{g} / \mathrm{mL}$ (DW $+\mathrm{KLX} 30 \mu \mathrm{g} / \mathrm{mL}$ ), diabetic wound treated with $\mathrm{KLX}$ oil $100 \mu \mathrm{g} / \mathrm{mL}$ (DW + KLX $100 \mu \mathrm{g} / \mathrm{mL}$ ), diabetic wound treated with $\mathrm{KLX}$ oil $300 \mu \mathrm{g} / \mathrm{mL}$ (DW + $\mathrm{KLX} 300 \mu \mathrm{g} / \mathrm{mL})$. All mice were anesthetized with $1 \%$ sodium pentobarbital $(0.1 \mathrm{~mL} / 10 \mathrm{~g})$, the back was shaved, and a skin wound with a diameter of $5 \mathrm{~mm}$ was made on the back with a skin punch. The NW and DW group were not treated with any drugs, and the rest groups were treated with $10 \mu \mathrm{L}$ of the corresponding drugs once a day at the wound site for 14 consecutive days. The wound healing of each mouse was recorded by taking pictures at the same focal length every day. Wound area is measured by Image Pro Plus software and the healing rate was calculated by the following formula: 
Healing rate $(\%)=\frac{(\text { Wound Area of Day } 1-\text { Wound Area of Day } \mathrm{N})}{\text { Wound Area of Day } 1} \times 100 \%$

\section{Angiogenesis on the subsurface of the skin}

Mice were sacrificed on the $7^{\text {th }}$ day after administration with corresponding drugs. Skin samples with a diameter of $1 \mathrm{~cm}$ around the wound were cut and the angiogenesis of the subsurface of the skin was recorded.

\section{Hematoxylin-eosin staining}

Mice were sacrificed on the $7^{\text {th }}$ day after administration with corresponding drugs. Skin samples with a diameter of $1 \mathrm{~cm}$ around the wound were cut and fixed with $10 \%$ paraformaldehyde for $48 \mathrm{~h}$. Then the tissue was embedded with paraffin and sliced into tissue sections with the thickness of $6 \mu \mathrm{m}$. Then tissue sections were processed into hematoxylin-eosin (HE) staining using HE Staining Kit. The nuclei was stained blue, and cytoplasm and connective tissue were stained red. Representative images of each group were taken by Leica microsystems (Solms, Germany).

\section{Masson staining}

Mice were sacrificed on the $7^{\text {th }}$ day after administration with corresponding drugs. Skin samples with a diameter of $1 \mathrm{~cm}$ around the wound were cut and fixed with $10 \%$ paraformaldehyde for $48 \mathrm{~h}$. Then the tissue was embedded with paraffin and sliced into tissue sections with the thickness of $6 \mu \mathrm{m}$. Then tissue sections were processed into Masson staining using Masson Staining Kit. Collagen fibers were stained blue or green. It is worth noting that the inherent collagen fibers were stained dark blue and the new collagen fibers were stained light blue. Representative images were taken by Leica microsystems (Solms, Germany).

\section{Immunohistochemistry}

Mice were sacrificed on the $7^{\text {th }}$ day after administration with corresponding drugs. Skin samples with a diameter of $1 \mathrm{~cm}$ around the wound were cut and fixed with $10 \%$ paraformaldehyde for 48 hours. Then the tissue was embedded with paraffin and sliced into tissue sections with the thickness of $6 \mu \mathrm{m}$. Then tissue sections were fixed on the glass slide pretreated with gelatin and washed twice in PBS for 5 minutes each. Then the tissue sections were treated with $0.1 \%$ triton X-100 penetration solution, washed with PBS for 30 minutes, and blocked at room temperature for 2 hours. Then the sections were incubated with aSMA primary antibody $(1: 1000)$ at $4{ }^{\circ} \mathrm{C}$ for 24 hours. After incubation, the sections were washed 6 times in PBS for 5 minutes each. Finally, the sections were incubated with secondary antibody for 2 hours. After incubation, the sections were washed 6 times in PBS for 5 minutes each, and sealed with glycerine sealant. Representative images were taken by Leica microsystems (Solms, Germany).

\section{Determination of hydroxyproline content}


Mice were sacrificed on the $3^{\text {th }}, 7^{\text {th }}, 11^{\text {th }}$ day after administration with corresponding drugs. Skin samples with a diameter of $1 \mathrm{~cm}$ around the wound were cut and the hydroxyproline content was measured. Determination was performed according to the requirements of the Hydroxyproline Assay Kit. The preparation method of test samples is as follows: skin tissue $10 \mathrm{mg}$ was hydrolyzed with hydrolysate 200 $\mu \mathrm{L}$ for 20 minutes in $95{ }^{\circ} \mathrm{C}$ metal bath. After cooling with running water, $10 \mu \mathrm{L}$ of indicator solvent was add to each sample. After shaking well, each sample was mixed with $200 \mu \mathrm{L} \mathrm{pH}$ solution A. Then $\mathrm{pH}$ solution $B$ was added dropwise to each sample tube and the sample solution was mixed thoroughly until the liquid becomes yellow green. Then the sample solution was diluted with $2 \mathrm{~mL}$ of deionized water. Transfer the diluted sample solution to a new tube and add an appropriate amount of activated carbon. After thorough mixing, the mixture was centrifuged at $3500 \mathrm{rpm}$ for 10 minutes and $200 \mu \mathrm{L}$ of supernatant was taken as the test sample. Then add the detection reagents 1,2 and 3 in the assay kit to the sample tubes respectively (deionized water served as a blank Control and $5 \mu \mathrm{g} / \mathrm{mL}$ hydroxyproline solution served as a standard control) and heat in a $60^{\circ} \mathrm{C}$ water bath for 15 minutes. After cooling, the mixture was centrifuged at $3500 \mathrm{rpm}$ for 10 minutes and the supernatant was taken and its absorbance at the wavelength of $550 \mathrm{~nm}$ was measured. The content of hydroxyproline was calculated according to the following equation:

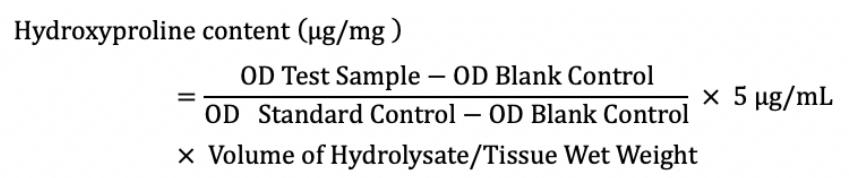

\section{Determination of cell proliferation}

Cell proliferation was determined by MTT assay according to previous method. Cultured HUVECs were seeded into 96-well cell culture plates at a density of $1 \times 10^{4} /$ well and divided into six groups: Control, AGEs, AGEs + DMSO, AGEs + KLX $1 \mu \mathrm{M}$, AGEs + KLX $10 \mu \mathrm{M}$ and AGEs + KLX $100 \mu \mathrm{M}$. Cells were pretreated with AGEs $100 \mu \mathrm{g} / \mathrm{mL}$ for 24 hours to establish a diabetic wound cell model and treated with the corresponding drugs for 30 minutes. In another experiment, cells were pretreated with PD173074 (FGFR inhibitor), PD153035 (EGFR inhibitor), ZM306416 (VEGFR inhibitor) and AG1296 (PDGFR inhibitor) for 12 hours to inhibit the activity of above receptors before incubating with KLX. Then the culture medium was discarded and MTT solution was added to each well in the dark. After incubating for 4 hours at $37^{\circ} \mathrm{C}$, the MTT-containing medium was discarded and $150 \mu \mathrm{L}$ of DMSO was added to each well. After shaking for 1 minute, the absorbance of each sample was detected at $490 \mathrm{~nm}$ with a microplate spectrophotometer (Bio-Tek, USA).

\section{Determination of cell migration}

Cell migration was determined by scratch assay. Cultured HUVEC was seeded into a 6-well cell culture plates at a density of $1 \times 10^{6} /$ well and divided into six groups: Control, AGEs, AGEs + DMSO, AGEs + KLX $0.1 \mu \mathrm{M}, \mathrm{AGEs}+\mathrm{KLX} 1 \mu \mathrm{M}$, AGEs + KLX $10 \mu \mathrm{M}$. A cell model of wound was prepared by drawing a straight line with a uniform width using the tip of a sterile pipette at the bottom of the culture plate. Then the cells 
were washed 3 times with PBS to remove cell debris. Except for the control group, cells in the other group were treated with AGEs $100 \mu \mathrm{g} / \mathrm{mL}$ and corresponding drugs. In another experiment, cells were pretreated with PD173074 (FGFR inhibitor) for 12 hours to inhibit the activity of FGFR before incubating with KLX. Cell migration in each well was recorded by taking photos at 0 hours, 12 hours and 24 hours after administration. The wound healing was calculated by Image-Pro-Plus Software.

\section{Determination of tubular structure formation}

The entire experiment should be performed at $4^{\circ} \mathrm{C}$. The equipment and reagents required for the experiment should be cooled in advance. Matrigel was placed at $4^{\circ} \mathrm{C}$ overnight to dissolve and diluted with FBS free PRIM-1640 medium in proportion of 1:1. Add $100 \mu \mathrm{L}$ of diluted Matrigel to the center well of a precooled laser confocal dishes, and transfer the dishes to the incubator for 30 minutes to allow Matrigel to solidify. At the same time, HUVECs cell suspension was prepared and mixed with corresponding drugs, and then uniformly seeded into laser confocal cultural dishes. Please note that air bubbles should be avoided throughout the process. Then the dished were incubated at $37^{\circ} \mathrm{C}$ and $5 \% \mathrm{CO}_{2}$ for 4 hours. The culture medium was discarded and the cells were stained with Calcein AM dye for 30 minutes. Then the cells were gently washed 3 times with PBS. The formation of the tubular structure was observed and recorded under Leica microsystems (Solms, Germany). Mesh number, node number and total tube length of the tubular structure was calculated by Image-Pro-Plus software.

\section{Western blot analysis}

Western blot analysis was performed according to the previous method [20]. Protein extraction experiments should be performed at $4^{\circ} \mathrm{C}$. Cells or tissues were lysed with ice-cold protein lysates (RIPA : phosphatase inhibitor : protease inhibitor $=100: 10: 1)$ and the lysed mixture was collected into a tube. The lysed mixture was sonicated and centrifuged at $13500 \mathrm{rpm}$ and $4^{\circ} \mathrm{C}$ for 15 minutes. The supernatant was collected and the protein concentration was measured. The protein samples were heated in a metal bath at $95^{\circ} \mathrm{C}$ for 10 minutes. Then the samples were cooled and subjected to gel electrophoresis. Protein samples were added to the gel wells for separation. After complete separation, protein was transferred to an NC membrane and blocked with $5 \%$ skim milk for 2 hours to block nonspecific bands. Then the NC membrane was washed with PBST on a shaker for 30 minutes and incubated with phospho-ERK $1 / 2$ and $\beta$-actin primary antibody $(1: 1000)$ at $4{ }^{\circ} \mathrm{C}$ overnight. Then the NC membrane was washed with PBST on a shaker for 30 minutes and incubated with the anti-HRP antibody (1:10000) for 50 minutes in the dark at room temperature. Then the NC membrane was washed with TBST on a shaker in the dark for 30 minutes. The expression of the target protein was detected by an infrared fluorescence scanning system, and the optical density integral value of the protein bands was analyzed by Odyssey 1.3 software.

\section{Molecular docking}

The discovery Studio 3.5 docking program was adopted here [21]. The preparation of protein structure (PDB code 3POZ) included adding hydrogen atoms, removing water molecules, and assigning Charm forcefield [22]. Goldscore was selected as the score function, and the other parameters were set as 
default. For each docking study, a total of 10 docking poses were retained. The root-mean square deviation (RMSD) between docking poses were calculated.

\section{Statistical analysis}

Data are expressed as mean \pm SEM. Data of each group was performed T-Test or Paired T-Test for statistical analysis. $p<0.05$ was considered statistically significant.

\section{Results}

\section{KLX accelerates the healing of diabetic wounds}

Wound healing of normal and diabetic mice was recorded for 14 consecutive days. Normal wounds (NW) and diabetic wounds (DW) were not treated with any medication. For the other diabetic wounds, the corresponding drugs were given once a day at the wound site for 14 consecutive days. Pictures of each wound were taken daily to record the healing process, and the wound area and healing rate were calculated using Image-Pro-Plus software. Compared with NW, the healing rate of DW was significantly reduced at each time point. Besides, the blank oil has no obvious improvement on the healing of diabetic wounds. However, daily administration of KLX significantly promotes the healing of diabetic wounds, especially the wounds treated with $\mathrm{KLX} 300 \mu \mathrm{g} / \mathrm{mL}$ in the first 7 days after trauma. Together all, $\mathrm{KLX}$ promotes the healing of diabetic wounds, and this effect is more pronounced in the middle and early stages of wound healing (Fig. 1).

\section{KLX promotes the formation of granulation tissue and new collagen in the wound area}

On the $7^{\text {th }}$ day after treatment with corresponding drugs, mice were sacrificed by injecting excessive pentobarbital sodium intraperitoneally. Skin samples with a diameter of $1 \mathrm{~cm}$ around the wound were cut off and fixed with $10 \%$ paraformaldehyde, followed by HE, Masson and immunohistochemical staining to determine the pathological changes of each wound. HE staining results showed that the granulation tissue formation and epidermal regeneration around wound area of NW were better than DW. Besides, DW showed obvious accumulation of inflammatory cells and fewer capillary blood vessels compared with NW. After administrated with KLX for 7 days, a large amount of granulation tissue and a more complete new epidermis were formed on the wound skin, indicating that KLX can significantly promote epidermal regeneration and granulation tissue formation in diabetic wounds. Besides, compared with DW, KLX obviously reduced inflammatory cells infiltrating into wound tissues and promoted neovascularization (Fig. 2a). In addition, Masson staining was performed to detect the effect of KLX on the formation of collagen fibers in diabetic wounds (the collagen fibers were stained blue, of which the native collagen was dark blue and the new collagen was light blue). Compared with NW, DW showed thinner skin tissues and fewer collagen fibers. After KLX treatment, the skin tissues became thickened and the new collagen fibers were increased significantly and arranged more tightly (Fig. 2c). In addition, a-SMA immunohistochemical staining was performed to detect the transformation of myofibroblasts in skin wounds. The transformation of myofibroblast contributes to secrete more collagen and increase the 
elasticity and tensile strength of wound skin. Results showed that the expression of a-SMA of DW was lower than NW, which indicated less myofibroblast transformation in DW. After KLX treatment, the expression of a-SMA was significantly increased, indicating the increase in myofibroblast transformation and collage formation (Fig. 2b). However, the blank oil did not have these beneficial effects. Besides, hydroxyproline assay kit was performed to detect the content of hydroxyproline in the wound with or without KLX treatment. Results showed that the content of hydroxyproline in KLX-treated wound was significantly higher than DW wound on the $3^{\text {th }}, 7^{\text {th }}$ and $11^{\text {th }}$ days after trauma (Fig. $2 d$ ).

\section{KLX promotes angiogenesis of diabetic wound skin}

Angiogenesis is a key factor in wound healing. Therefore, we examined the effect of KLX on angiogenesis in both in vivo and in vitro studies. Firstly, in vivo study, mice were sacrificed by injecting excessive pentobarbital sodium intraperitoneally on the $7^{\text {th }}$ day after the corresponding drugs was administrated. Skin samples with a diameter of $1 \mathrm{~cm}$ around the wound were cut off. Angiogenesis on the subcutaneous surface of the wound was observed and recorded. The results showed that more new blood vessels were formed on the subcutaneous surface of NW skin. Compared with NW, the number of blood vessels on the subcutaneous of DW skin was significantly reduced. KLX significantly promoted the formation of new blood vessels on the subcutaneous surface of DW skin, while blank oil did not have this promotive effect (Fig. 3a). Next, we further verified the role of KLX in promoting angiogenesis at the in vitro level. Firstly, HUVECs were treated with AGEs $100 \mu \mathrm{g} / \mathrm{mL}$ to inhibit its proliferation to establish an cell model of diabetic wounds, and the effect of KLX on the inhibition of HUVEC proliferation by AGEs was detected by MTT assay. The results showed that AGEs significantly inhibited the proliferation of HUVECs. KLX $10 \mu M$ and $100 \mu \mathrm{M}$ significantly reduced the inhibitory effect of AGEs on HUVECs proliferation in a dosedependent manner, while DMSO (solvent of KLX) had no significant effect on the inhibitory effect of

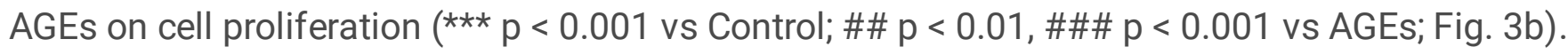
Secondly, the effect of KLX on migration ability of HUVEC was detected by scratch test. The results showed that HUVEC migration was significantly inhibited after treating cells with AGEs for 12 and 24 hours. KLX $10 \mu \mathrm{M}$ significantly alleviated the inhibitory effect of AGEs on HUVECs migration, while DMSO had no significant effect on AGEs-inhibited cell migration ( $* p<0.05$, $* \star p<0.01$ vs Control; $\# p<0.05$, \#\# $p<0.01$ vs AGEs; Fig. 3c-d). Finally, the effect of KLX on angiogenesis ability of HUVECs was detected by tubule formation experiments using Matrigel as a carrier. The results showed that AGEs significantly reduced the tubule formation ability of HUVECs. Compared with the control group, the number of nodes, meshes and the total length of the tubular structures of AGEs-treated cells were significantly reduced (* $p$ $<0.05,{ }^{* \star} p<0.01$ vs. control, $n=3$ ). However, after KLX treatment, the tube-forming ability of HUVECs was significantly enhanced, and the number of nodes, meshes and total length of the tubular structures were significantly increased ( $* \star p<0.01$, ${ }^{\star \star *} p<0.001$ vs. AGEs, $n=3$; Fig. 3e-h).

\section{KLX activated ERK1/2 signaling pathway}

Activation of ERK1/2 signaling pathway can stimulate the proliferation and survival of vascular endothelial cells, thereby promoting angiogenesis [23]. Therefore, effect of KLX on the phosphorylation of 
ERK $1 / 2$ in wound tissues on the $7^{\text {th }}$ day after trauma was examined by Western blot experiments. The results showed that there was no statistical difference in the protein abundance of phospho-ERK $1 / 2$ in DW compared with NW, while KLX significantly increased the protein abundance of phospho-ERK1/2 of DW ( $p<0.05, n=3$; Fig. 4a). The same results were also obtained in HUVECs. After HUVECs was treated with $100 \mu \mathrm{M} \mathrm{KLX}$ for 30 minutes, the protein abundance of phospho-ERK1/2 increased significantly $(p<$ $0.05, n=3$; Fig. $4 b)$. These results indicate that $K L X$ promotes angiogenesis by activating the ERK $1 / 2$ signaling pathway.

\section{Effect of KLX in promoting proliferation of vascular endothelial cells is related to FGFR activity}

Cytokines and growth factors regulate angiogenesis and promote wound healing process. Studies have shown that the dysregulation of cytokines and growth factors, including degradation of growth factors and downregulation of growth factor receptors, affects angiogenesis of skin and wound healing. Studies have found that growth factors such as VEGF, FGF, PDGF, EGF, etc, are significantly reduced in diabetic patients, which may be an important factor causing wound healing disorders. Therefore, these growth factors and their receptors can be molecular targets for the treatment of diabetic wound. In this study, we used inhibitors of these growth factor receptors to verify whether the angiogenic effects of KLX were achieved by affecting these growth factor receptors. Firstly, the effects of EGFR inhibitor PD153035, FGFR inhibitor PD173074, VEGFR inhibitor ZM306416 and PDGFR inhibitor AG1296 on the proliferative effect of KLX on HUVECs were determined by MTT assay. Results showed that AGEs significantly inhibited HUVECs proliferation, while KLX reversed the inhibitory effect of AGEs on HUVEC proliferation, which was similar to the results of previous studies. In addition, PD173074, ZM306416 and AG1296 inhibited the promotion of HUVEC proliferation by KLX, of which PD173074 had the most significant inhibitory effect. The EGFR inhibitor PD153035 had no inhibitory effect on the proliferative effect of KLX. These results indicate that the FGFR inhibitor PD173074 significantly reversed the proliferative effects of KLX, suggesting that the proliferation of KLX may be mediated by the activation of FGFR (Fig. $5 \mathrm{a}$ ).

\section{Molecular Docking simulation of KLX and FGFR}

To explore the potential binding of KLX and FGFR, a molecular docking was performed by Discovery Studio 3.5 to view the potential interactions. As shown in Figure $5 \mathrm{~b}, \mathrm{KLX}$ can subtly occupy the binding pocket. The a-hydroxyl group of KLX can form a hydrogen bond with the Glu562 side chain located in the hinge region of kinase. Two carbonyl groups of the succinate side chain of KLX form two conservative hydrogen bonds with the Gly 485 residue of FGFR. Additionally, the anthraquinone core of KLX is deeply embedded in the hydrophobic pocket, including Leu484, Leu630 and Val492 residues, forming a hydrophobic interaction. The above results provided a potential structural basis for KLX as a potential FGFR agonist.

\section{KLX promotes angiogenesis by activating the FGFR/ERK1/2 signaling pathway}

The results of molecular docking simulations suggest that KLX can activate FGFR and downstream signaling pathways by acting as a ligand for FGFR. Therefore, we investigated the effect of FGFR 
inhibitor PD173074 on the pharmacological action of KLX in phosphorylating ERK1/2 and promoting angiogenesis. Firstly, the effect of PD173074 on KLX phosphorylating ERK1/2 was detected by Western blot experiment. The result showed that KLX significantly increased the protein abundance of phosphoERK $1 / 2$ to $2.02 \pm 0.30$ folds of Control group, while PD173074 significantly reversed KLX's promotion of ERK1/2 phosphorylation (Fig. 5c). Besides, PD173074 significantly reversed KLX's promotion of HUVECs migration and tube formation (Fig. 7d-i). These results suggest that FGFR inhibitor significantly reverse the pharmacological effects of KLX on ERK1/2 phosphorylation and HUVEC proliferation, migration and tubular structure formation. Therefore, we can speculate that KLX promotes angiogenesis by activating the FGFR/ERK signaling pathway.

\section{Discussion}

In our previous studies, we found the vascular endothelium protecting property of KLX and confirmed its pharmacological effects of lowering blood pressure [20]. In this study, we explored the effect of KLX in promoting diabetic wound healing and the underlying molecular mechanisms. In vivo study, KLX significantly accelerated the healing of skin wound in diabetic mice. Pathological studies of the wound skin showed that KLX promoted the formation of granulation tissue and new collagen, facilitated angiogenesis and reduced infiltration of inflammatory cells in diabetic wounds. In vitro studies confirmed that KLX promoted HUVEC proliferation, migration, tubular structure formation and ERK $1 / 2$ phosphorylation, further proving KLX's role in promoting angiogenesis. Molecular docking simulations show that KLX has potential binding sites to amino acid residues of FGFR, suggesting that KLX may act as a ligand for FGFR and activate it. Besides, inhibition of FRFR significantly reversed the effects of KLX on phosphorylating ERK1/2 and promoting angiogenesis. These data demonstrate the therapeutic effect of KLX on diabetic wounds, and KLX exerts this effect by activating FGFR/ERK1/2 mediated angiogenesis.

Abnormal blood glucose handling ability of diabetic patients complicates and prolongs the wound healing process [24]. Under normal circumstances, wound healing is a dynamic process regulated by multiple factors. Normal wounds can heal completely in $2 \sim 3$ weeks, while the healing process of diabetic wounds may be extended to more than 8 weeks [25]. Hyperglycemia severely affects all stages of wound healing, including hemostasis, inflammation, proliferation, and remodeling. Diabetic wounds is characterized with a continuous inflammatory phase, mature granulation tissue formation obstacle, decreased collagen content, thinned skin tissue and reduced wound tensile strength [26-27]. In this study, we established a type 1 diabetes model with mouse by injecting STZ intraperitoneally. Although type 2 diabetes accounts for about $90 \%$ of total diabetic patients, type 1 diabetes animal models are more widely used in the study of diabetic wounds, because of its uncomplicated preparation, high modeling rate, and consistent abnormal glucose metabolism process with type 2 diabetes [28-29]. Our research results confirmed that KLX accelerated the healing speed of diabetic wounds. Pathological analysis of skin samples further indicated that KLX significantly reduced the infiltration of inflammatory cells, promoted the regeneration of granulation tissue, new collagen and new blood vessels around diabetic 
wounds. These effects of KLX contribute to accelerate the transition of wound tissue from the inflammatory stage to the proliferative stage, thereby promoting tissue regeneration and wound healing.

The tardy healing of diabetic wounds involves complex molecular mechanisms. Vascular damage and microangiopathy of the wound skin cause ischemia and hypoxia in the wound. Sustained ischemia and hypoxia could induce an endoplasmic reticulum stress response and promote the production of proinflammatory mediators, which is the main reason why the diabetic wounds continue to be in the inflammatory stage [30]. New blood vessels can effectively fill the damaged area of skin, increase the blood and oxygen supply to the damaged skin, and correct the ischemic and hypoxic conditions of wound tissues [31-32]. Therefore, promoting angiogenesis is a key factor in accelerating skin wound healing. In this study, we explored the effects of KLX on angiogenesis in vivo and in vitro. Firstly, in animal studies, KLX significantly increased the density of blood vessels on the subsurface of the skin of diabetic wounds. In addition, in vitro study, HUVEC was pretreated with AGEs to establish a cell model of diabetic wounds. AGEs are stable end products produced by condensation, rearrangement, pyrolysis, and oxidative modification between free amino groups of proteins, amino acids, lipids, or nucleic acids and aldehyde groups of reducing sugars [33]. Under normal physiological conditions, AGEs accumulate slowly in our body with age increasing. However, chronically high or fluctuating blood glucose accelerates AGEs formation in diabetes patients (or pre-diabetes people), resulting in the rapid accumulation of AGEs in the internal environment [34]. AGEs attach to healthy cells, joints, organs, skin, and various other systems, and accelerate the development of diabetes and diabetic complications directly or indirectly. AGEs affect normal physiological functions of cells and hinder diabetic wound healing through inducing the production of cytokines and inflammatory factors, inhibiting the generation of collagen, and inducing excessive immune response $[35,36]$. Therefore, AGEs have been used widely as a stimulant for preparing in vitro models of diabetic wounds $[37,38]$. Our results confirmed that AGEs significantly inhibit the proliferation, migration, and tubular structure formation of HUVECs, whereas KLX significantly reverses the inhibitory effect of AGEs. Based on these results, our research revealed that KLX has the function of promoting angiogenesis and antagonizing the harmful effect of AGEs. These effects provide a preliminary explanation for its role in accelerating wound healing.

Pro-angiogenic factors, such as VEGF, FGF, EGF, PDGF, are involved in the regulation of angiogenesis. The binding of theses growth factors with their receptors can activate downstream signaling pathways and promote the proliferation, migration and tubular structure formation of vascular endothelial cells [39-40]. Studies have shown that in diabetic wounds, the production and release of these pro-angiogenic growth factors are reduced, their degradation is increased, or their receptors are down-regulated, leading to the dysfunction of growth factors in the wound area and preventing wound healing process from entering the proliferative stage [41]. VEGF is the most effective angiogenic factor. VEGF can increase the capillary density of diabetic wounds, improve the blood perfusion and metabolism of injured tissues, and provide nutrients for repairing cells and wound tissues by activating VEGFR2 $[42,43]$. FGF is also a key regulator of angiogenesis. FGFR is a kind of tyrosine receptor. The combination of FGF and FGFR causes FGFR activation and autophosphorylation, thereby initiating intracellular signal transduction and promoting angiogenesis [44, 45]. PDGF-BB is a hypoxia-regulated gene product of VEGFA, which promotes wound 
healing at all stages [46]. Currently, the growth factor-based drugs that have been used in clinical treatment are PDGF-BB and bFGF. However, their application has certain limitations. In acute wounds, various cells can promote the restoration of skin barrier function under the action of growth factor drugs. Unlike ordinary acute wounds, diabetic wounds exhibit a longer inflammatory period. Inflammatory cells infiltrate the wound surface to form a proteolytic environment, leading to the hydrolysis of growth factor drugs. In addition, pro-inflammatory factors and chemokines at the wound site are continuously upregulated, which can also lead to the degradation and disconnection of growth factors and cytokines, thereby inhibiting their functions [18]. In addition, it is worth noting that the healing ratio of diabetic wound is still less than $50 \%$ with the intervention of so many existing drugs. Therefore, there is still an urgent need for research and development of drugs that can upregulate related growth factor receptors to activate downstream proliferation signaling pathway and promote the healing of chronic wounds. Based on this point, this study further verified whether KLX's role in promoting angiogenesis is related to the activity of growth factor receptors. Firstly, we treated HUVEC with inhibitors of EGFR, FGFR, PDGFR and VEGFR to observe the effect of inhibiting the function of these receptors on KLX's promotion of cell proliferation. The results showed that except for EGFR inhibitor, the other three inhibitors significantly inhibited the effect of KLX on promoting HUVEC proliferation, and FGFR inhibitor was the most efficacious. Secondly, the results of the molecular docking simulation showed that there were multiple hydrogen bonds between KLX and FGFR amino acid residues. Hydrogen bond is the most important way of intermolecular bonding, and it is also an important way for drugs to interact with proteins. This result further demonstrates the potential of KLX to interact with FGFR. Thirdly, FGFR inhibitor also inhibit the

role of KLX in promoting ERK1/2 phosphorylation and angiogenesis, further indicating that KLX promotes angiogenesis by activating FGFR and its downstream ERK signaling pathways (Fig. 6).

\section{Conclusion}

In conclusion, our results confirm the therapeutic effect of KLX in diabetic wounds. KLX promotes angiogenesis and the healing of diabetic wounds by activating FGFR and its downstream ERK signaling pathway. Current study indicates that KLX is a new candidate compound for treating diabetic wounds. Further study needs to ensure the effectiveness and safety of KLX preparations in the treatment of diabetic wounds in order to promote the application of KLX in clinical treatment.

\section{Abbreviations}

AGEs: advanced glycated end products; EGF: epidermal growth factor; EGFR: epidermal growth factor receptor; ERK1/2: extracellular signal-regulated kinase 1/2; FGF: fibroblast growth factor; FGFR: fibroblast growth factor receptor; HUVEC: human umbilical vein endothelial cells; PDGF-BB: platelet-derived growth factor-BB; PDGFR: platelet-derived growth factor receptor; VEGF: vascular endothelial growth factor; VEGFA: vascular endothelial growth factor A; VEGFR: vascular endothelial growth factor receptor; VEGFR2: vascular endothelial growth factor receptor 2; STZ: Streptozocin. 


\section{Declarations}

\section{Ethics approval and consent to participate}

This study was carried out in accordance with the recommendations of US National Institutes of Health $(\mathrm{NIH})$ guidelines for the care and use experimental animals. The protocol was approved by Ethics Committee of College of Pharmacy, Harbin Medical University.

\section{Consent for publication}

Not applicable.

\section{Availability of data and materials}

The datasets used and/or analyzed during the current study are available from the corresponding author on reasonable request.

\section{Competing interests}

The authors declare that they have no competing interests.

\section{Funding}

This work was supported by National Natural Science Foundation of China (No. 81903608, No. 81870259, No. 81730012), National Science and Technology Major Project of the Ministry of Science and Technology of China (No. 2018ZX09735005), NSFC-FRQS project (No. 2019-2021).

\section{Authors' contributions}

YaZ and BY designed and supervised the research; $Y x Z$ analyzed the research data and prepared the original draft; $X W$ conducted the animal experiments; $S Y$ and $X S$ contributed to cell culture and molecular biology experiment; CC and SW completed pathology and morphology experiments. All authors approved the submitted version of manuscript.

\section{Acknowledgements}

We appreciate the science and technology park of Harbin medical university for providing us with required laboratories and instruments. We are also thankful for the other support from Department of Pharmacology of Harbin Medical University.

\section{References}

1. International Diabetes Federation. IDF Diabetes Atlas. 8th ed. Brussels: International Diabetes Federation; 2017. 
2. International Diabetes Federation. - What is diabetes. https://www.idf.org/aboutdiabetes/what-isdiabetes.html. Accessed 5 Sep 2019.

3. Sharp A, Clark J. Diabetes and its impact on wound healing. Nurs Stand. 2011;25:41-7.

4. Okonkwo UA, DiPietro LA. Diabetes and wound angiogenesis. Int J Mol Sci. 2017;18(7):1419.

5. Hicks CW, Selvarajah S, Mathioudakis N, Sherman RE, Hines KF, Black JH 3rd, et al. Burden of infected diabetic foot ulcers on hospital admissions and costs. Ann Vasc Surg. 2016;33:149-58.

6. Lim JZ, Ng NS, Thomas C. Prevention and treatment of diabetic foot ulcers. J R Soc Med. 2017;110(3):104-9.

7. Patel S, Srivastava S, Singh M, Singh D. Mechanistic insight into diabetic wounds: Pathogenesis, molecular targets and treatment strategies to pace wound healing. Biomed Pharmacother. 2019;112:108605.

8. Singh MR, Saraf S, Vyas A, Jain V, Singh D. Innovative approaches in wound healing: trajectory and advances, Artif. Cell Nanomed Biotechnol. 2013;41:202-12.

9. Alavi A, Sibbald RG, Mayer D, Goodman L, Botros M, Armstrong DG, et al. Dabetic foot ulcers part I. Pathophysiology and prevention. J Am Acad Dermatol. 2014;1:e1-1e16.

10. Galkowska H, Wojewodzka U, Olszewski WL. Chemokines, cytokines, and growth factors in keratinocytes and dermal endothelial cells in the margin of chronic diabetic foot ulcers. Wound Repair Regen. 2006;14:558-65.

11. Davis FM, Kimball A, Boniakowski A, Gallagher K. Dysfunctional wound healing in diabetic foot ulcers: new crossroads. Curr Diab Rep. 2018;18(1):2.

12. Galiano RD, Tepper OM, Pelo CR, Bhatt KA, Callaghan M, Bastidas N, et al. Topical vascular endothelial growth factor accelerates diabetic wound healing through increased angiogenesis and by mobilizing and recruiting bone marrow-derived cells. Am J Pathol. 2004;164:1935-47.

13. Qi M, Zhou Q, Zeng W, Wu L, Zhao S, Chen W, et al. Growth factors in the pathogenesis of diabetic foot ulcers. Front Biosci (Landmark Ed). 2018;23:310-7.

14. Shaikh-Kader A, Houreld NN, Rajendran NK, Abrahamse $H$. The link between advanced glycation end products and apoptosis in delayed wound healing. Cell Biochem Funct. 2019;37(6):432-42.

15. Peppa M, Stavroulakis P, Raptis SA. Advanced glycoxidation products and impaired diabetic wound healing. Wound Repair Regen. 2009;17(4):461-72.

16. Everett E, Mathioudakis N. Update on management of diabetic foot ulcers. Ann N Y Acad Sci. 2018;1411(1):153-65.

17. Zhang L, Yin H, Lei X, Lau JNY, Yuan M, Wang X, et al. A systematic review and meta-analysis of clinical effectiveness and safety of hydrogel dressings in the management of skin wounds. Front Bioeng Biotechnol. 2019;7:342.

18. Laiva AL, O'Brien FJ, Keogh MB. Innovations in gene and growth factor delivery systems for diabetic wound healing. J Tissue Eng Regen Med. 2018 Jan;12(1):e296-312. 
19. Kulprachakarn K, Ounjaijean S, Wungrath J, Mani R, Rerkasem K. Micronutrients and natural compounds status and their effects on wound healing in the diabetic foot ulcer. Int J Low Extrem Wounds. 2017;16(4):244-50.

20. Zhao YX, Zhu JX, Liang HF, Yang S, Zhang YN, Han WN, et al. Kang Le Xin reduces blood pressure through inducing endothelial-dependent vasodilation by activating the AMPK-eNOS pathway. Front Pharmaco. 2020;10:1548.

21. Wu G, Robertson DH, Brooks CL, Vieth M. Detailed analysis of grid-based molecular docking: A case study of CDOCKER-A CHARMm-based MD docking algorithm. J Comput Chem. 2003;24:1549-62.

22. Aertgeerts K, Skene R, Yano J, Sang BC, Zou H, Snell G, et al. Structural analysis of the mechanism of inhibition and allosteric activation of the kinase domain of HER2 protein. J Biol Chem. 2011;286(21):18756-65.

23. Ishikura K, Misu H, Kumazaki M, Takayama H, Matsuzawa-Nagata N, Tajima N, et al. Selenoprotein P as a diabetes-associated hepatokine that impairs angiogenesis by inducing VEGF resistance in vascular endothelial cells. Diabetologia. 2014;57(9):1968-76.

24. Kodner C, Anderson L, Pohlgeers K. Glucose management in hospitalized patients. Am Fam Physician. 2017;96(10):648-54.

25. Anisha BS, Biswas R, Chennazhi KP, Jayakumar R. Chitosan-hyaluronic acid/nano silver composite sponges for drug resistant bacteria infected diabetic wounds. Int J Biol Macromol. 2013;62:310-20.

26. Boniakowski AE, Kimball AS, Jacobs BN, Kunkel SL, Gallagher KA. Macrophage-mediated inflammation in normal and diabetic wound healing. J Immunol. 2017;199(1):17-24.

27. Zhao R, Liang H, Clarke E, Jackson C, Xue M. Inflammation in chronic wounds. Int J Mol Sci. 2016;17(12):2085.

28. Bagheri M, Mostafavina A, Abdollahifar M, Amini A, Ghoreishi S, Chien S, et al. Combined effects of metformin and photobiomodulation improve the proliferation phase of wound healing in type 2 diabetic rats. Biomed Pharmacother. 2020;123:109776.

29. Zhao M, Zhou J, Chen YH, Yuan L, Yuan MM, Zhang XQ, et al. Folic acid promotes wound healing in diabetic mice by suppression of oxidative stress. J Nutr Sci Vitaminol (Tokyo). 2018;64(1):26-33.

30. Haemmerle M, Keller T, Egger G, Schachner H, Steiner CW, Stokic D, et al. Enhanced lymph vessel density, remodeling, and inflammation are reflected by gene expression signatures in dermal lymphatic endothelial cells in type 2 diabetes. Diabetes. 2013;62(7):2509-29.

31. Trinh NT, Yamashita T, Ohneda K, Kimura K, Salazar GT, Sato F, et al. Increased expression of EGR-1 in diabetic human adipose tissue-derived mesenchymal stem cells reduces their wound healing capacity. Stem Cells Dev. 2016;25(10):760-73.

32. Lepantalo M, Biancari F, Tukiainen E. Never Amputate without consultation of a vascular surgeon. Diabetes Metab Res Rev. 2000;16(Suppl):27-32.

33. Cano Sanchez M, Lancel S, Boulanger E, Neviere R. Targeting oxidative stress and mitochondrial dysfunction in the treatment of impaired wound healing: a systematic review. Antioxidants (Basel). 2018;7(8):98. 
34. Fishman SL, Sonmez H, Basman C, Singh V, Poretsky L. The role of advanced glycation end-products in the development of coronary artery disease in patients with and without diabetes mellitus: a review. Mol Med. 2018;24(1):59.

35. Hu H, Jiang H, Ren H, Hu X, Wang X, Han C. AGEs and chronic subclinical inflammation in diabetes: disorders of immune system. Diabetes Metab Res Rev. 2015;31(2):127-37.

36. Berlanga-Acosta J, Schultz GS, López-Mola E, Guillen-Nieto G, García-Siverio M, Herrera-Martínez L. Glucose toxic effects on granulation tissue productive cells: the diabetics' impaired healing. Biomed Res Int. 2013;2013:256043.

37. Jin H, Zhang Z, Wang C, Tang Q, Wang J, Bai X, et al. Melatonin protects endothelial progenitor cells against AGE-induced apoptosis via autophagy flux stimulation and promotes wound healing in diabetic mice. Exp Mol Med. 2018;50(11):1-15.

38. Yuan YF, Das SK, Li MQ. Vitamin D ameliorates impaired wound healing in streptozotocin-induced diabetic mice by suppressing endoplasmic reticulum stress. J Diabetes Res. 2018;2018:1757925.

39. Ishihara J, Ishihara A, Fukunaga K, Sasaki K, White MJV, Briquez PS, et al. Laminin heparin-binding peptides bind to several growth factors and enhance diabetic wound healing. Nat Commun. 2018;9(1):2163.

40. Barrientos S, Stojadinovic O, Golinko MS, Brem H, TomicCanic M. Growth factors and cytokines in wound healing. Wound Repair Regen. 2008;16:585-601.

41. Crovetti G, Martinelli G, Issi M, Barone M, Guizzardi M, Campanati B, et al. Platelet gel for healing cutaneous chronic wounds. Transfus Apher Sci. 2004;30:145-51.

42. Jude EB, Eleftheriadou I, Tentolouris N. Peripheral arterial disease in diabetes-a review. Diabet Med. 2010;27:4-14.

43. Zhou K, Ma Y, Brogan MS. Chronic and non-healing wounds: the story of vascular endothelial growth factor. Med Hypotheses. 2015;85:399-404.

44. Katoh M. Therapeutic targeting FGF signaling network in human diseases. Trends Pharmacol Sci. 2016;37(12):1081-96.

45. Zheng Z, Kang HY, Lee S, Kang SW, Goo B, Cho SB. Up-regulation of fibroblast growth factor (FGF) 9 expression and FGF-WNT/ $\beta$-catenin signaling in laser-induced wound healing. Wound Repair Regen. 2014;22(5):660-5.

46. Kaltalioglu K, Coskun-Cevher S, Tugcu-Demiroz F, Celebi N. PDGF supplementation alters oxidative events in wound healing process: A time course study. Arch Dermatol Res. 2013;305:415-22.

\section{Figures}


a

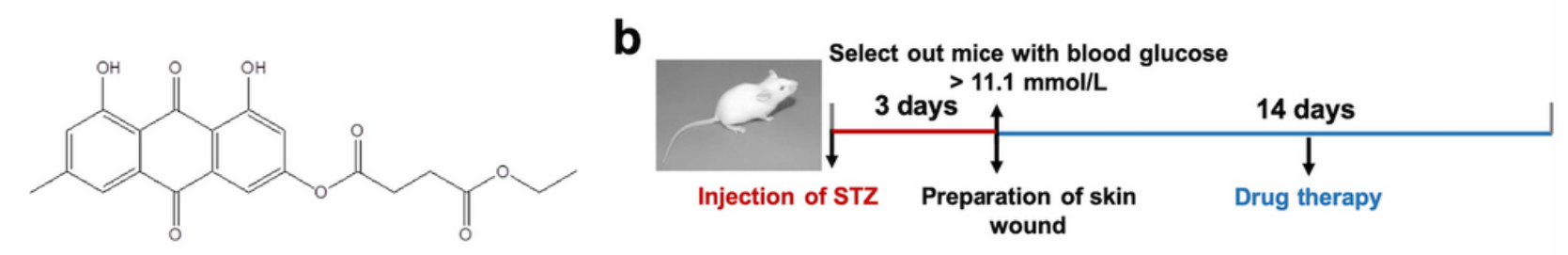

C

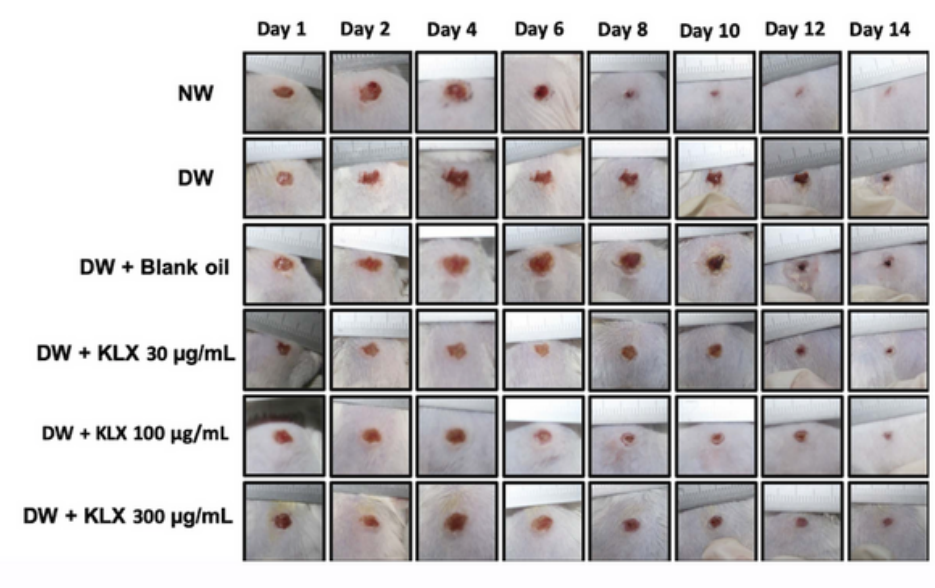

d

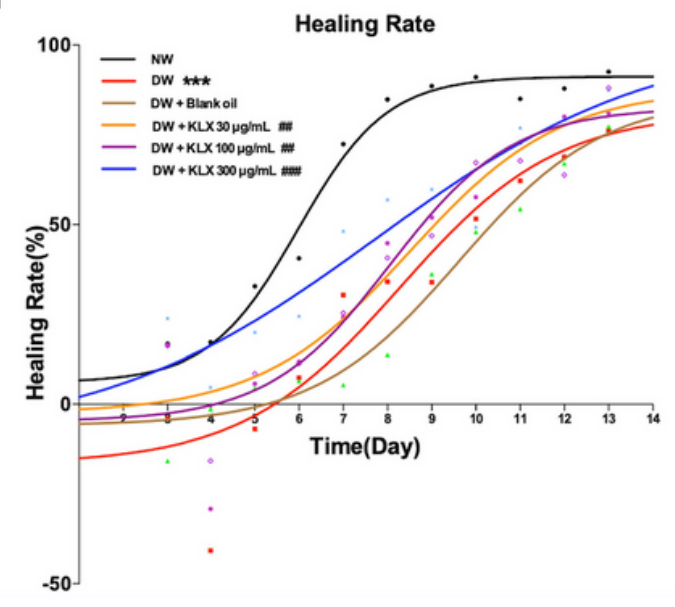

\section{Figure 1}

KLX accelerates the healing of diabetic wounds. a Chemical structure of KLX. b Flow diagram of experiment. c Representative images of wound healing for 14 consecutive days. NW represents nondiabetic mice without any treatment at the wound site; DW represents diabetic mice without any medication at the wound site; DW + Blank oil represents diabetic wounds treated with $10 \mu \mathrm{L}$ of blank oil daily; $\mathrm{DW}+\mathrm{KLX} 30 \mu \mathrm{g} / \mathrm{mL}$ represents diabetic wounds treated with $10 \mu \mathrm{L}$ of $\mathrm{KLX}(30 \mu \mathrm{g} / \mathrm{mL})$ daily; DW + $\mathrm{KLX} 100 \mu \mathrm{g} / \mathrm{mL}$ represents diabetic wounds treated with $10 \mu \mathrm{L}$ of $\mathrm{KLX}(100 \mu \mathrm{g} / \mathrm{mL})$ daily; DW + KLX 300 $\mu \mathrm{g} / \mathrm{mL}$ represents diabetic wounds treated with $10 \mu \mathrm{L}$ of $\mathrm{KLX}(300 \mu \mathrm{g} / \mathrm{mL})$ daily. $\mathrm{d}$ Fitting curve of diabetic wound healing after different drug treatments. ${ }^{* \star} p<0.001$ compared with NW; \#\# $p<0.01$, \#\#\# $\mathrm{p}<0.001$ compared with DW; $\mathrm{n}=6$.

原

\section{Figure 2}

$\mathrm{KLX}$ promotes granulation tissue, new collagen and new blood vessels formation at the wound. a HE staining of wound skin, $n=3$. b Immunohistochemical staining of $a-S M A$ in the wound skin, $n=3$. $c$ Masson staining of wound skin, $n=3$. d Content of hydroxyproline in wound skin, ${ }^{\star} p<0.05,{ }^{*} * 0<0.001$ compared with NW; \#\# $p<0.01$, \#\#\# $p<0.001$ compared with DW; $n=5$. 
a

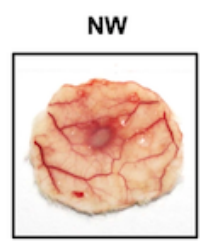

DW

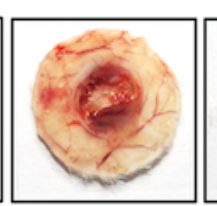

DW

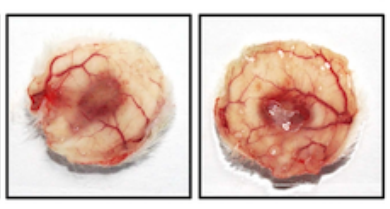

C

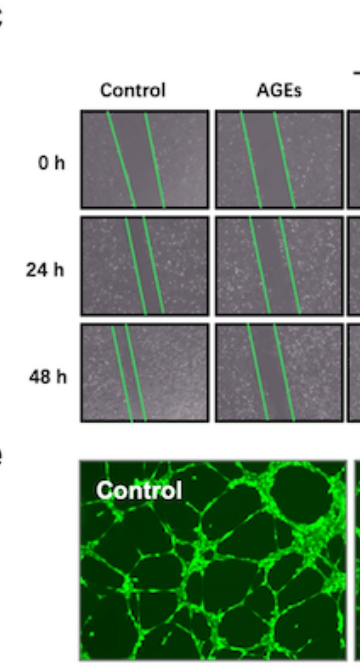

f

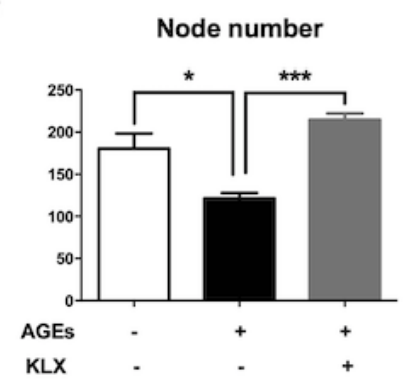

AGES
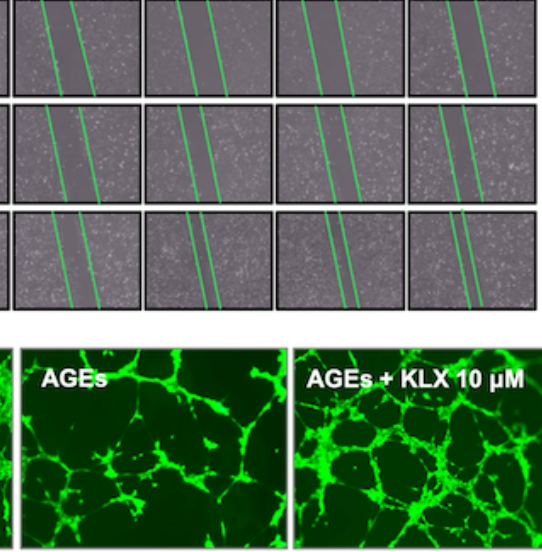

g

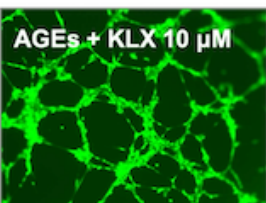

g

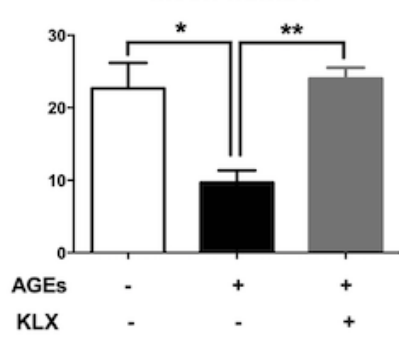

b Cell proliferation

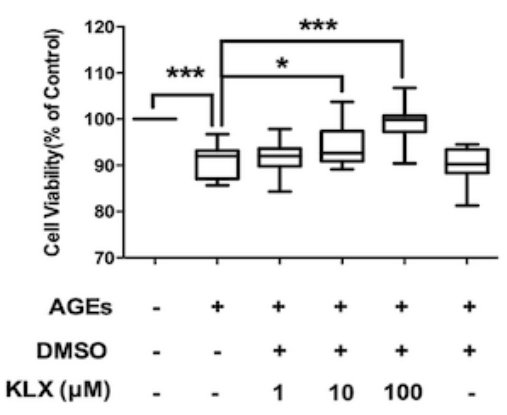

d

Healing Rate

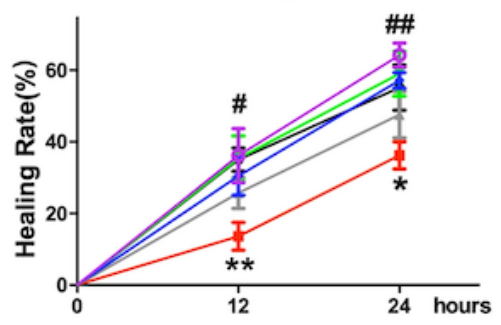

h

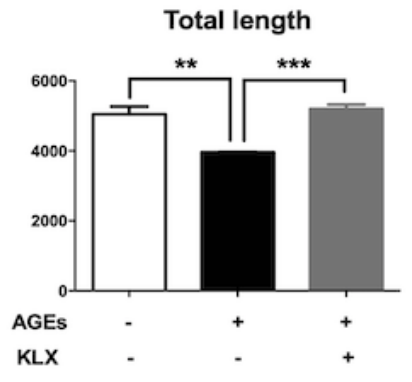

\section{Figure 3}

KLX promotes angiogenesis of diabetic wound skin. a Representative image show that the formation of new blood vessels on the subcutaneous surface of wound skin, $n=3$. b Effect of KLX on the inhibition of HUVECs proliferation by AGEs ( $* p<0.05, * \star * p<0.001 ; n=4)$. c Representative images show that the effect of KLX on the inhibition of HUVECs migration by AGEs. $d$ Statistical graph of cell migration in each group ( $* p<0.05$, ** $p<0.01$ vs. Control; $\# p<0.05$, \#\# $p<0.01$ vs. AGEs; $n=4$ ). e Representative images show that the effect of KLX on the inhibition of angiogenesis by AGEs. $f$ Statistical graph of the number of nodes in each group of tubular structures. g Statistical graph of the number of meshes in each group of tubular structures. $\mathrm{h}$ Statistical graph of the number of total length in each group of tubular structures ( $\star p<0.05, * * p<0.01, * \star * p<0.001 ; n=3)$. 
a
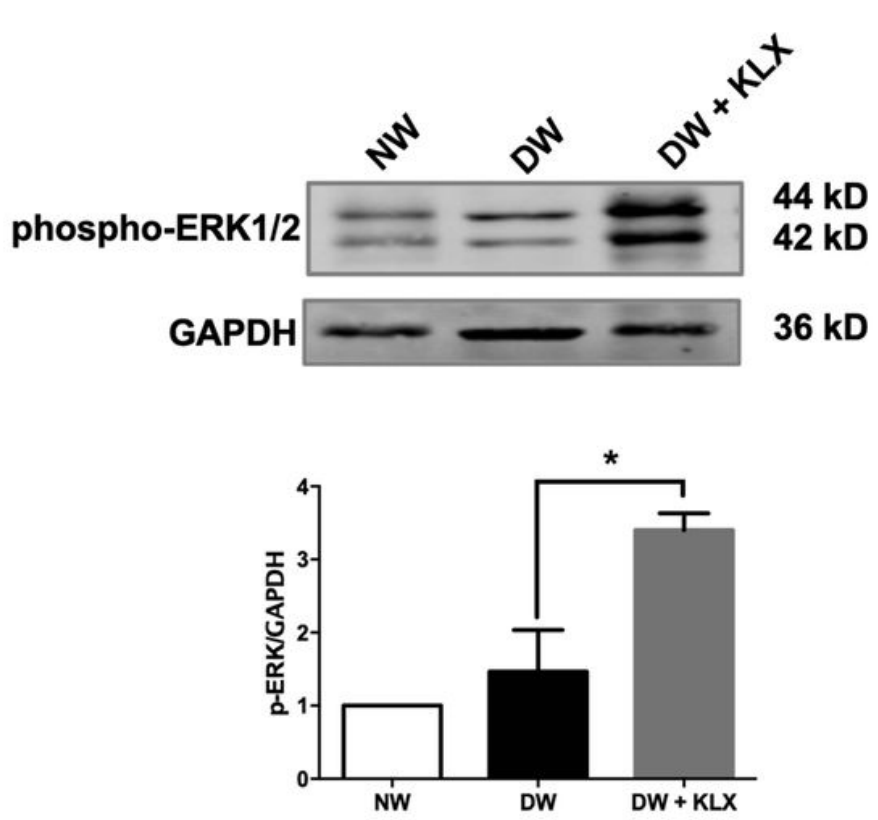

b

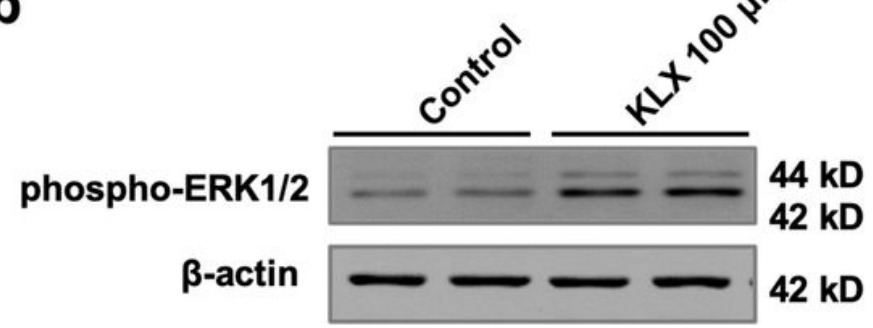

\section{Figure 4}

KLX phosphorylates ERK1/2 in diabetic wounds and HUVECs. a Effect of KLX on the phosphorylation of ERK1/2 in the skin tissue of wound on the 7th day after trauma. ${ }^{*} p<0.05, n=3$. b Effect of KLX on the phosphorylation of ERK1/2 in HUVECs. * $p<0.05, n=3$. 

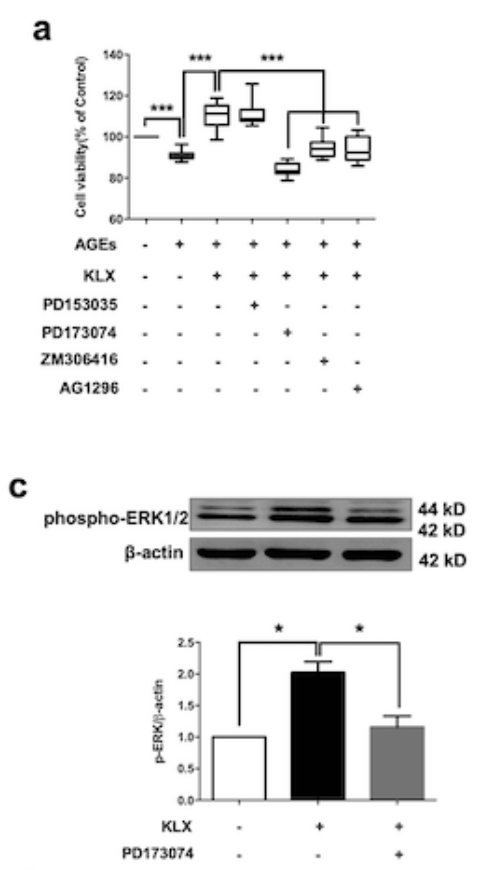
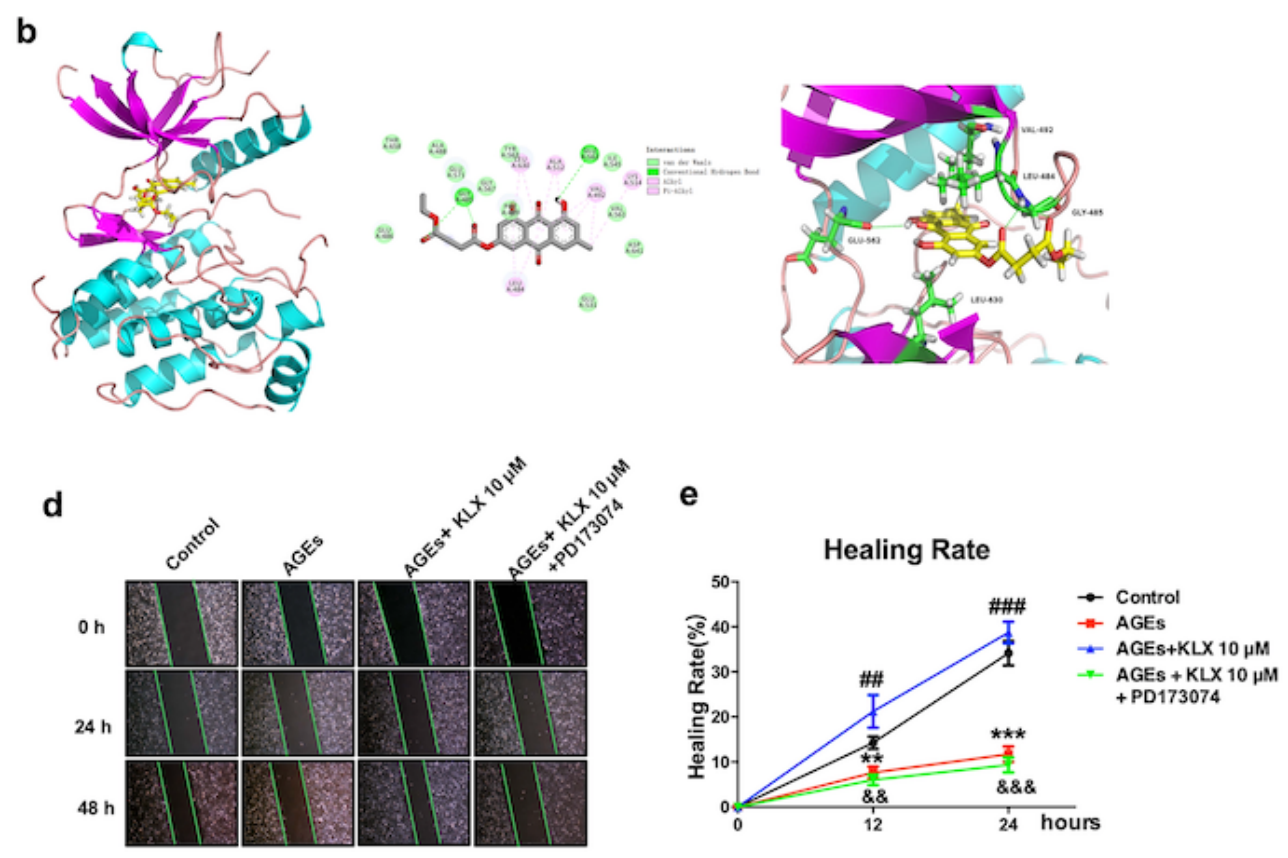

f
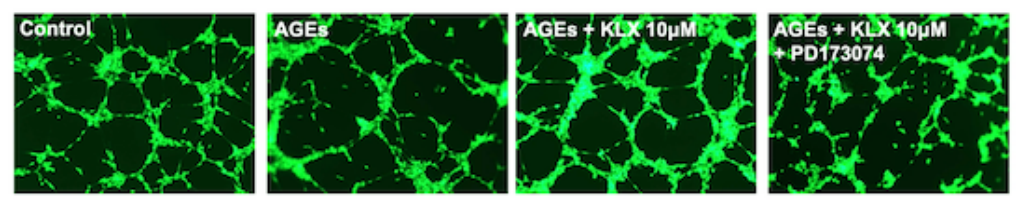

g

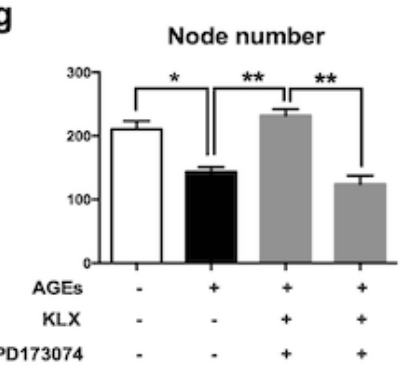

h

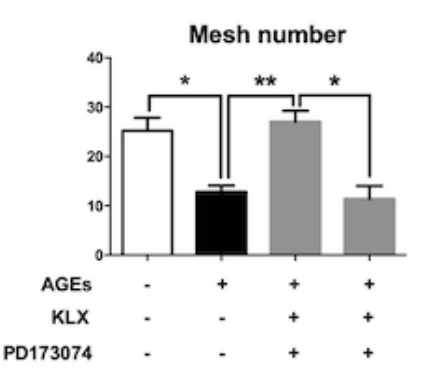

i

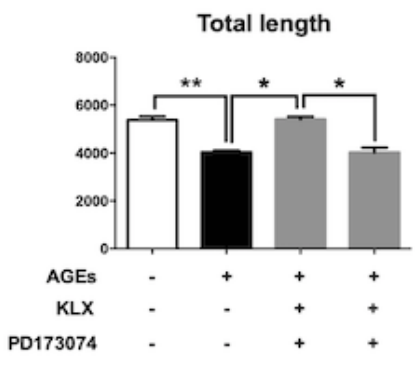

\section{Figure 5}

KLX promotes angiogenesis by activating the FGFR/ERK1/2 signaling pathway. a Effects of EGFR inhibitor PD153035, FGFR inhibitor PD173074, VEGFR inhibitor ZM306416 and PDGFR inhibitor AG1296 on the proliferation of HUVEC by KLX; $* \star \star ~ p<0.001, n=6$. b Molecular docking of KLX and FGFR. $c$ Effects of FGFR inhibitor PD173074 on KLX promoting ERK1/2 phosphorylation, $p<0.05, n=3$. $d$ Representative images show the effect of FGFR inhibitor PD173074 on KLX promoting HUVEC migration, $\mathrm{n}=6$. e Statistical graphs of cell migration rate show that the effect of FGFR inhibitor PD173074 on KLX

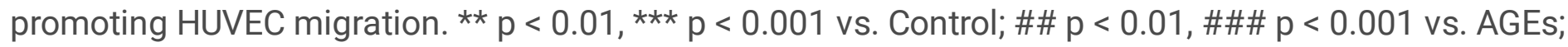
$\& \& p<0.01, \& \& \& p<0.001$ vs. AGEs $+\mathrm{KLX} 10 \mu \mathrm{M} ; \mathrm{n}=6$. f Representative images show that the effect of FGFR inhibitor PD173074 on KLX's promotion of tube formation, $n=3$. g Statistical graph of the number of nodes in tube structure in the tube formation experiment. $\mathrm{h}$ Statistical graph of the number of the 
meshes in tube structure in the tube formation experiment. i Statistical graph of total length of tube structure in the tube formation experiment. ${ }^{*} p<0.05,{ }^{*} p<0.01, n=3$.
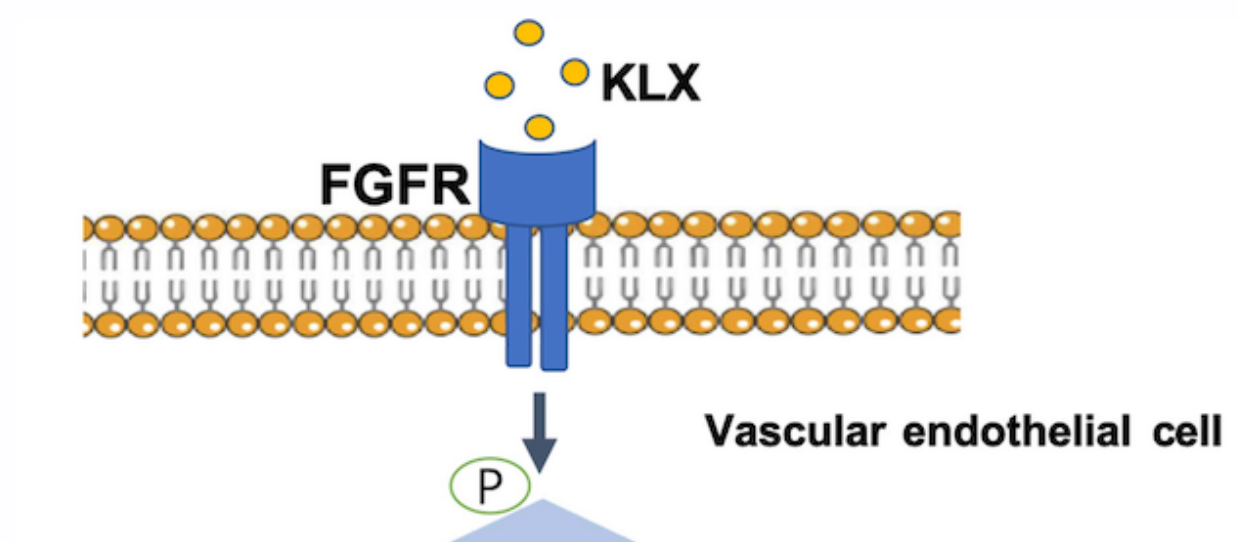

\section{ERK1/2}

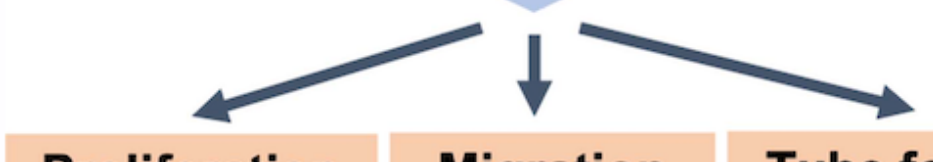

\section{\begin{tabular}{l|l|l} 
Proliferation & Migration & Tube formation
\end{tabular}}

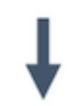

Angiogenesis
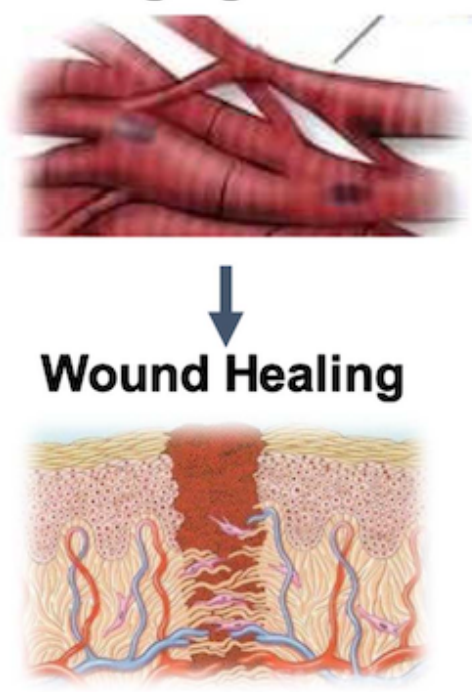

Figure 6

Schematic diagram of the proposed signaling mechanisms by which KLX promotes angiogenesis and wound healing. 